\title{
The role of neutral anions in ionic liquid as solvent media for the reactivity and stereoselectivity towards asymmetric Michael addition reaction of n-pentanal with B- nitrostryrene catalyzed by L-Proline
}

\begin{abstract}
Michael addition reactions of n-pentanal with $\beta$-nitrostyrene in achiral and chiral ionic liquids catalyzed by 1-proline were studied. Results indicate anion plays an important role as weak coordination properties in the reactivity and stereoselectivity towards Michael product. 1-Butyl3-methylimidazolium bis(trifluoromethylsulfonyl)imide ([Bmim][NTf2]) ionic liquid was found to be the best solvent media with a high yield (up to 90\%) and a high diastereomeric ratio (syn/anti: 91/9), with moderate enantioselectivity (38\% ee) among ten ionic liquids tested. The ionic liquid has been reusable over five numbers of cycles.
\end{abstract}

Keyword : Anion; Cation; Chiral; Enantioselective; Ionic liquid; 1-proline; Michael addition 